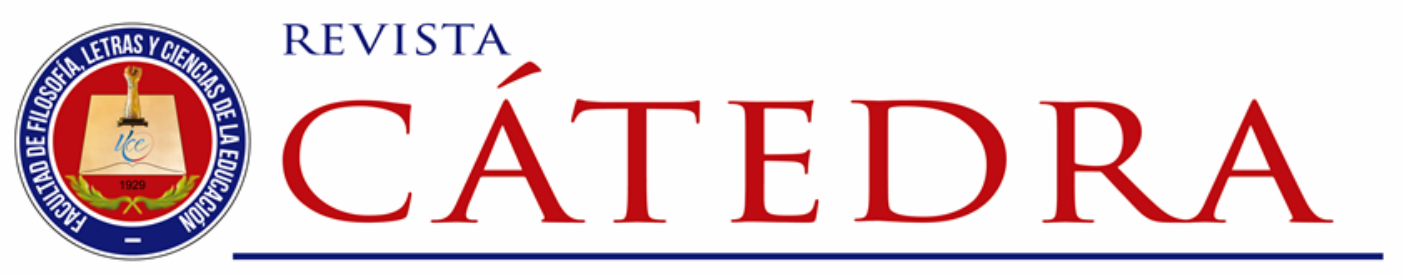

\title{
Inclusión y territorialidad en la nivelación general para el examen Ser Bachiller en el Ecuador
}

\section{Inclusion and territoriality in the general leveling for the Ser Bachiller exam in Ecuador}

\author{
Oswaldo Haro-Jácome \\ Universidad Central del Ecuador, Quito, Ecuador \\ oharo@uce.edu.ec \\ https://orcid.org/0000-0001-6387-9591 \\ Héctor Simbaña-Cabrera \\ Universidad Central del Ecuador, Quito, Ecuador \\ hasimbana@uce.edu.ec \\ https://orcid.org/0000-0002-9802-5470
}

Juan Aguilar-Paoquiza

Escuela Superior Politécnica del Chimborazo

juan.aguilar@espoch.edu.ec https://orcid.org/0000-0001-8882-5717

(Recibido: 09/03/2020; Aceptado: 13/04/2020; Versión final recibida: 07/05/2020)

Cita del artículo: Haro-Jácome, O., Simbaña-Cabrera, H. y Aguilar-Poaquiza, J. (2020). Inclusión y territorialidad en la nivelación general para el examen Ser Bachiller en el Ecuador. Revista Cátedra, 3(2), 17-34.

\section{Resumen}

El artículo describe la inclusión: por género, etnia, discapacidad; y por territorialidad provincial, cantonal e institucional de bachilleres participantes en el Curso de Nivelación General, segundo proceso del año 2019, ejecutado por la Secretaría de Educación Superior, Ciencia, Tecnología e Innovación (SENESCYT), en convenio con la Empresa Pública UCE. El tratado puntualiza la situación de derechos de la juventud que se capacita para rendir el 
examen Ser Bachiller. La investigación se fundamenta en el enfoque cuantitativo no experimental, los hechos se abordan desde la perspectiva transversal y su profundidad es exploratoria-descriptiva. Los instrumentos de campo fueron: la encuesta en línea aplicada mediante la plataforma de la Universidad Central del Ecuador, aplicada a 7665 bachilleres, de los cuales contestaron 4073 que se constituyó en la muestra casual no probabilística. Las notas para observar participación-aprobación se extrajeron de las aulas virtuales por dominio de la plataforma. Se descubrió que bachilleres desertores y reprobados superan levemente a aquellos promocionados. En inclusión por género, la promoción de mujeres es muy superior a varones y otros géneros; por etnias los mestizos superan ampliamente la participación y aprobación. No se reportaron casos de bachilleres con discapacidad en el curso. En territorialidad, los bachilleres de la región litoral superan al resto de provincias y cantones de otras regiones; mientras que por instituciones, los planteles educativos públicos, regentados por el Estado, tienen mayor participación.

\section{Palabras clave}

Bachilleres, educación superior, inclusión, nivelación académica, territorialidad.

\section{Abstract}

The article describes inclusion: by gender, ethnicity, disability; and by provincial, cantonal and institutional territoriality of high school graduates participating in the General Leveling Course, second process of the year 2019, executed by the Ministry of Higher Education, Science, Technology and Innovation (SENESCYT), in agreement with the Public Company UCE. The treaty clarifies the situation of youth rights that are trained to take the Ser Bachiller exam (Be Bachelor). The research is based on the non-experimental quantitative approach, the facts are approached from the transectional perspective and its depth is exploratory-descriptive. The field instruments were: the online survey applied through the http://www.filosofia-uce.com/ platform of the Central University of Ecuador, applied to 7,665 high school students, of which 4,073 answered that it was the casual sample not probabilistic. The notes to observe participation-approval were extracted from the virtual classrooms by domain of the platform. It was discovered that defending and failed high school graduates slightly exceed those promoted. In gender inclusion, the promotion of women is far superior to men and other genders; by ethnic groups the mestizos (half blood) widely exceed the participation and approval. No cases of high school graduates with disabilities were reported in the course. In territoriality, high school graduates from the coastal region surpass the rest of the provinces and cantons of other regions. While by institutions the public educational establishments, run by the State, have greater participation.

\section{Keywords}

High school graduates, higher education, inclusion, academic leveling, territoriality.

\section{Introducción}

Las políticas de postulación a las instituciones de Educación Superior públicas del Ecuador (IES) como: universidades, politécnicas e institutos se implementó como proyecto piloto en el año 2011 y de forma oficial y obligatoria en el año 2012. Las normas que rigen el ingreso a las IES son: la Constitución 2008, la Ley Orgánica de Educación Superior (LOES) y el Sistema Nacional de Nivelación y Admisión (SNNA). La modalidad de ingreso mediante 
exámenes tiene ya nueve años de vigencia y ha sido muy controversial, debido a factores como: derechos, inclusión, territorialidad, interculturalidad e incluso el tipo de examen.

El ingreso a las IES públicas mediante la aprobación examen, en su ejecución pasó por dos etapas bastante diferenciadas. En una primera etapa el Examen Nacional para la Educación Superior (ENES) a partir del año 2012 y luego el Ser Bachiller desde el 2017.

Uno de los aspectos de mayor controversia es la concepción inclusiva o meritocrática del ingreso a las IES. Sus principios orientadores planeados por la SENESCYT, constantes en el artículo 3) del Reglamento del Sistema Nacional de Nivelación y Admisión (SNNA) 2019, dispone que son los: "(...) méritos, igualdad de oportunidades, y libertad de elección de carrera o carreras e institución” (SENESCYT, 2019, pág. 2).

Los principios del SNNA son fácilmente rebatibles, ya que la meritocracia según afirmación de Cociña (2013), una “(...) sociedad meritocrática -que se corresponde con la visión utópica de una sociedad de mercado- es una sociedad que, al menos en principio, no reduce -ni mucho menos elimina- los niveles actuales de desigualdad o de miseria" (pág. 4). La admisión al sistema educativo superior ecuatoriano, dadas las condiciones muy dispares en el reparto de la riqueza, ha causado innumerables dificultades en el acceso a la universidad y la carrera de interés de cada bachiller, en similares oportunidades.

Los datos para el análisis de campo se extrajeron de la encuesta de los factores asociados del Curso de Nivelación General, mayo-julio 2019, facilitado por la Empresa Pública UCE a bachilleres ecuatorianos, en convenio con la SENESCYT. Este curso fue facilitado por la Unidad de Vinculación con la Sociedad de la Facultad de Filosofía, Letras y Ciencias de la Educación.

El propósito del estudio es analizar los factores de inclusión y territorialidad en la participación-aprobación de bachilleres que asistieron al proceso de Nivelación General para el examen Ser Bachiller. El período corresponde al segundo proceso, mayo-julio 2019, según la planificación anual de la Subsecretaría de Nivelación (SENESCYT-Empresa Pública UCE, 2019).

Las preguntas que se proponen indagar, a la luz del estado de la cuestión y del dato empírico, son:

1. ¿De qué forma la capacitación para el examen Ser Bachiller que ofrece el Estado a bachilleres ecuatorianas/os, identifica y atiende las diferencias de inclusión y territorialidad, para reducir la exclusión de la educación superior, especialmente a jóvenes en situación de ruralidad y periferia de ciudades? (Inclusión Educativa, 2016),

2. ¿Cómo se evidencia equidad territorial: geográfica, social e individual, de bachilleres en la nivelación virtual ofrecida por la SENESCYT, ¿para el examen de ingreso a las IES públicas ecuatoriana? (Aceves, 1997, pág. 286).

Finalmente, mediante el tratado se busca explicar la contribución que brinda la nivelación general de la SENESCYT a la disminución de la amplia brecha en el puntaje del examen Ser Bachiller. Sobre todo, incluyendo a pueblos y nacionalidades, como forma de democratizar el ingreso al Sistema de Educación Superior, con puntajes competitivos que faciliten el acceso a IES y carreras de su vocación e interés personal.

El artículo tiene la siguiente estructura: en primer lugar, la introducción con unas aproximaciones conceptuales básicas, el objetivo, preguntas de investigación. En segundo 
lugar, las definiciones de las variables de estudio: nivelación general, inclusión, territorialidad. En tercer lugar, el proceso metodológico. Posteriormente constan los resultados, discusión, y conclusión.

\subsection{Nivelación general}

La nivelación general de los bachilleres que se interesan por postular a las IES públicas ecuatorianas, así como, lograr una beca nacional o internacional para estudios superiores, está reglamentada en varios cuerpos legales. Las normas que determinan la nivelación son básicamente la Constitución 2008, y más concretamente la Ley Orgánica de Educación Superior (LOES), que en la aplicación del Art. 81, prevé "un Sistema Nacional de Nivelación y Admisión, que garantiza la igualdad de oportunidades en el acceso, permanencia, movilidad y en el egreso" (Escuela Politécnica Nacional, 2019, pág. 1).

Hay dos procesos de nivelación en el ingreso a la educación superior regulado por el Art. 3 del Reglamento de la LOES que expresa la nivelación, tomando en cuenta la heterogeneidad en la formación del bachillerato y/o las características de las carreras universitarias" (SNNA, 2011, pág. 10).

En primer lugar, se ejecuta la nivelación general, que es la capacitación gratuita bajo la responsabilidad de la SENESCYT (anteriormente por las IES), a bachilleres que no lograron obtener un cupo en una o más rendiciones del examen Ser Bachiller. Este curso se desarrolla dos veces por año; una para las regiones costa e insular y otra para las regiones sierra y amazonia. Es justamente en la nivelación general, primer período del año 2019 que se realizó el estudio de campo.

En segundo lugar, la nivelación de Carrera, que es un curso de inducción para bachilleres que superaron ya el Ser Bachiller, postularon y fueron aceptados a una Carrera Universitaria. Esta capacitación se operativiza por áreas del conocimiento de la UNESCO y está a cargo de las IES.

\subsection{Inclusión educativa}

La inclusión es un factor esencial para todo proceso de prestación de servicios en el Ecuador, más en la evaluación de saberes con fines de acceso a derechos. La inclusión educativa es un principio de la Ley Orgánica de Educación Intercultural, que en el Artículo 11 literal v. establece:

La equidad e inclusión aseguran a todas las personas el acceso, permanencia y culminación en el Sistema Educativo. Garantiza la igualdad de oportunidades a comunidades, pueblos, nacionalidades y grupos con necesidades educativas especiales y desarrolla una ética de la inclusión con medidas de acción afirmativa y una cultura escolar incluyente en la teoría y la práctica en base a la equidad, erradicando toda forma de discriminación. (Asamblea Nacional, 2011, pág. 11).

El principio de inclusión encierra al conjunto de la sociedad ecuatoriana. Todo bachiller que se interese por ingresar a la IES, debe tener oportunidad sin discrimen por su origen, condiciones sociales, culturales y peor aún por diferencias físicas, formas de aprender o de salud, es decir, no hay requisitos previos para ejercer derechos. Al respecto, sostiene Leiva (2013), que: "si bien la educación inclusiva presta especial atención a grupos vulnerables y marginados, su fin es desarrollar al completo el potencial de todo individuo y no sólo el de grupos específicos" (pág. 7).

Licencia Creative Commons Atribución 4.0 Internacional (CC BY 4.0)

Revista Cátedra, 3(2), pp. 17-34, mayo-agosto 2020. e-ISSN:2631-2875

https://doi.org/10.29166/10.29166/catedra.v3i2.2053 
El principio de meritocracia declarado por el reglamento del SNNA es completamente contradictorio a la inclusión social y particularmente a la educativa, porque mientras la primera pone a toda la sociedad en una misma condición educativa, lo cual es una quimera en una sociedad extremadamente heterogénea entre sectores socioeconómicos; la segunda reconoce claramente las diferencias entre los sectores sociales, pero aboga por procesos permanentes que reconozcan esas diferencias, para beneficio equitativo de todas y todos, en este caso, en el ingreso a la universidad.

\subsection{Territorialidad}

La categoría territorialidad actualmente es muy utilizada en los sistemas de planificación social, especialmente en los proyectos de intervención para mejoramiento de las condiciones de vida de sectores comunitarios. Fundamentalmente se enfoca en el ser humano y al espacio geográfico, como un todo dialéctico que interactúa en la sociedad con efectos culturales, económicos e incluso medioambientales en la ruralidad. Farrell, Trillón, y Soto (1999) testifican que, "el enfoque territorial induce la integración de la institucionalidad pública y privada vinculada al desarrollo rural y el empoderamiento de los actores locales participantes, aspectos fundamentales para la apropiación del proceso; además, tiene un efecto multiplicador sobre otros territorios" (pág. 10).

Otro concepto muy extendido establece que: "El enfoque territorial en el desarrollo rural significa un cambio de perspectiva: de lo sectorial a lo multisectorial, promoviendo el análisis multidimensional (elementos económicos, políticos, sociales y ambientales, entre otros)" (Villalobos, 2015, pág. s/p). Es que justamente en ese espacio geográfico, esencialmente humano y armónico, donde sucede la vida, ocurren esas multiformes relaciones y formas de organización-acción-colaboración, para que la sociedad perviva y se transforme en el tiempo.

Al analizar a la nivelación general en la variable territorialidad, "el territorio en esta perspectiva debe ser considerado más allá de su dimensión de dominio, integrado en una concepción cultural y social, además de la administrativa y de gestión que tradicionalmente viene predominando" (García y Muñoz, 1999, pág. 181). El enfoque de territorialidad es un factor multidimensional que está presente en la atención al derecho de ingreso a las IES ecuatorianas, más allá de lo puramente geográfico.

\subsection{Inclusión y territorialidad en la nivelación general}

La inclusión educativa y de territorialidad en la nivelación general facilitada por la SENESCYT, está aún muy lejos de acoger a los bachilleres en condiciones óptimas pese a que se han tomado importantes medidas institucionales últimamente, es decir, con políticas de atención para el ingreso eficaz a las IES. Sobre inclusión en la educación superior, particularmente en la nivelación general, Pérez-Castro (2019) sostiene que se debería buscar:

La ampliación de las oportunidades educativas para todos aquellos colectivos en situación de vulnerabilidad, que han quedado excluidos del sistema escolar (...) por razones de desigualdad social (pobreza, ruralidad, género, etnia y discapacidad); causas institucionales (planes de acción, condiciones de la oferta, estrategias institucionales, recursos, entre otros); o por factores personales subjetivos (creencias, valores y actitudes) (pág. 149). 
Los principios de meritocracia, equidad e igualdad de oportunidades que orientan al examen de ingreso a la educación superior en Ecuador, tanto en el ENES como al Ser Bachiller, contradicen con la inclusión, porque universalizan un mismo examen y sus consecuencias para todos. Mientras que la inclusión implica procesos diferenciados, para atender las particularidades y déficit de muchos bachilleres, mediante test diferenciados, contextualización del examen y acción afirmativa.

Para atender la inclusión y la territorialidad como derechos humanos de bachilleres, Haro (2018) recomienda que debe actuarse mediante "una relación institucional fuerte entre el Ministerio de Educación y la SENESCYT para diseñar y aplicar políticas claras en la transición educativa de los bachilleres a la universidad, reconociendo los aspectos culturales, históricos y socioeconómicos de la sociedad ecuatoriana" (pág. 298). La política pública de postulación universitaria implica la coparticipación de todos los entes estatales relacionados con el derecho de la juventud, proceso que está en ciernes aún.

El proceso de transición del bachillerato a la universidad, es complejo por sí mismo, ya que los adolescentes están superando una etapa de crisis personal. Lamentablemente los jóvenes no cuentan con una orientación profesional en el colegio y sobre todo su proceso educativo, en la práctica, es muy diferenciado entre lo urbano y rural, así como entre la centralidad y la periferia de la ciudad. Las condiciones socioeconómicas son mayoritariamente de pobreza en las familias. Los aspectos señalados vienen negando permanentemente el acceso a la educación superior.

\section{Metodología}

El estudio se desarrolló bajo los principios del paradigma metodológico cuantitativo, un diseño no experimental. El abordaje del fenómeno fue transversal y el análisis fue exploratorio-descriptivo por ser un fenómeno nuevo en el contexto ecuatoriano. El tratamiento estadístico se realizó con la aplicación del programa Excel para organizar los datos, luego se sometió al paquete SPSS para el análisis de las variables.

La data se extrajo de la encuesta de factores asociados aplicada al inicio del curso y de las estadísticas de aprobación de la nivelación general, modalidad virtual, alojados en la plataforma de la UCE, http://www.filosofia-uce.com/, a cargo de la Dirección de Tecnologías de la Información y Comunicaciones (DTIC), entre los meses de mayo y junio de 2019. La encuesta se aplicó a los bachilleres como requisito para su matrícula en mayo, mientras que las estadísticas de aprobación se obtuvieron de la base de datos en junio. El tratamiento de la información fue un componente obligatorio de los términos de referencia (TDR) con la SENECSYT. Las notas para observar la promoción se tomaron de las aulas virtuales de los cinco dominios alojados en la plataforma.

La población de estudio se constituyó por 7.665 bachilleres beneficiarios del curso, previamente reportados por la Subsecretaría de Nivelación de la SENESCYT. La muestra efectiva que proporcionó los datos para el análisis empírico fue de 4.073 bachilleres, quienes participaron efectivamente en la nivelación. La técnica de muestreo fue de conveniencia, misma que según sostienen Otzen y Manterola (2017), en donde se aplica por “(...) la conveniente accesibilidad y proximidad de los sujetos para el investigador" (pág. 30).

El criterio de representatividad muestral es relativo, pues la muestra supera al $50 \%$ de la población, lo cual permite generalizar los resultados a la población objetivo, sin embargo, un factor limitante es que la selección muestral no fue al azar.

Licencia Creative Commons Atribución 4.0 Internacional (CC BY 4.0)

Revista Cátedra, 3(2), pp. 17-34, mayo-agosto 2020. e-ISSN:2631-2875

https://doi.org/10.29166/10.29166/catedra.v3i2.2053 


\section{Resultados y discusión}

El cuadro 1 resume la matrícula, movimiento y promoción de bachilleres en el Curso de Nivelación General ejecutado por la Faculta de Filosofía (FFLCE) de la Universidad Central del Ecuador (UCE). Los 4073 bachilleres que accedieron a la plataforma y son participantes en el curso en todo el país constituyen el $100 \%$ de beneficiarios efectivos, se constituye en la muestra del estudio. De este gran total, 2184 jóvenes, correspondiente al 53.62\% no terminaron sus evaluaciones en la plataforma virtual, luego de haber realizado ya aportes y evaluaciones, por lo tanto, se categorizan como retirados y superan la mitad de los beneficiarios. En criterio de García (2007), las "causales de abandono (...) de una oferta educativa online (son) los materiales del curso (...), poco tiempo (...) para la realización de la tarea, las condiciones tecnológicas (...) y las comunicaciones con el tutor como elemento facilitador y orientador" (pág. 12). Concluyen el curso 1889 bachilleres, correspondiente al $46,38 \%$, menos de la mitad de aquellos bachilleres retirados.

\begin{tabular}{|c|c|c|c|c|c|c|c|}
\hline \multirow[b]{2}{*}{$\begin{array}{l}\text { Modalidad } \\
\text { Virtual }\end{array}$} & \multirow{2}{*}{$\begin{array}{l}\text { Matrícula } \\
\text { Reportados } \\
\text { por SENESCYT } \\
\text { a la UCE }\end{array}$} & \multirow[b]{2}{*}{$\begin{array}{l}\text { Matrícula } \\
\text { UCE }\end{array}$} & \multicolumn{2}{|c|}{$\begin{array}{l}\text { Movimiento de } \\
\text { Bachilleres }\end{array}$} & \multicolumn{3}{|c|}{ Promoción Final } \\
\hline & & & $\begin{array}{l}\text { No acceden } \\
\text { Plataforma }\end{array}$ & $\begin{array}{l}\text { Acceden } \\
\text { Plataforma }\end{array}$ & Retirados & Reprobados & Aprobados \\
\hline \multirow[t]{3}{*}{$\begin{array}{l}\text { Totales de } \\
\text { inicio }\end{array}$} & 7.665 & 7.665 & 3.592 & 4.073 & 2.184 & 433 & 1.456 \\
\hline & ataje de avance & $100 \%$ & $46.9 \%$ & $53.1 \%$ & & & \\
\hline & \multicolumn{3}{|c|}{ Porcentajes de promoción (final) } & $100 \%$ & $53.62 \%$ & $10 \%$ & $35.75 \%$ \\
\hline
\end{tabular}

Cuadro 1. Matrícula, movimiento y promoción de bachilleres en el curso

La figura 1 sistematiza el acceso de bachilleres a la plataforma de la UCE, en donde de 7665 bachilleres efectivamente matriculados, interactúan en aulas virtuales. En primer lugar, se describe la evolución de su participación en el curso, desde la matrícula hasta la promoción, donde $3592(46,9 \%)$ jóvenes no ratificaron su matrícula en la plataforma de nivelación de la Universidad Central del Ecuador. Consecuentemente, 4073 bachilleres se beneficiaron de la capacitación efectiva, correspondiente al 53.1\% del total de bachilleres matriculados, representando una cantidad superior de quienes no se matricularon. 


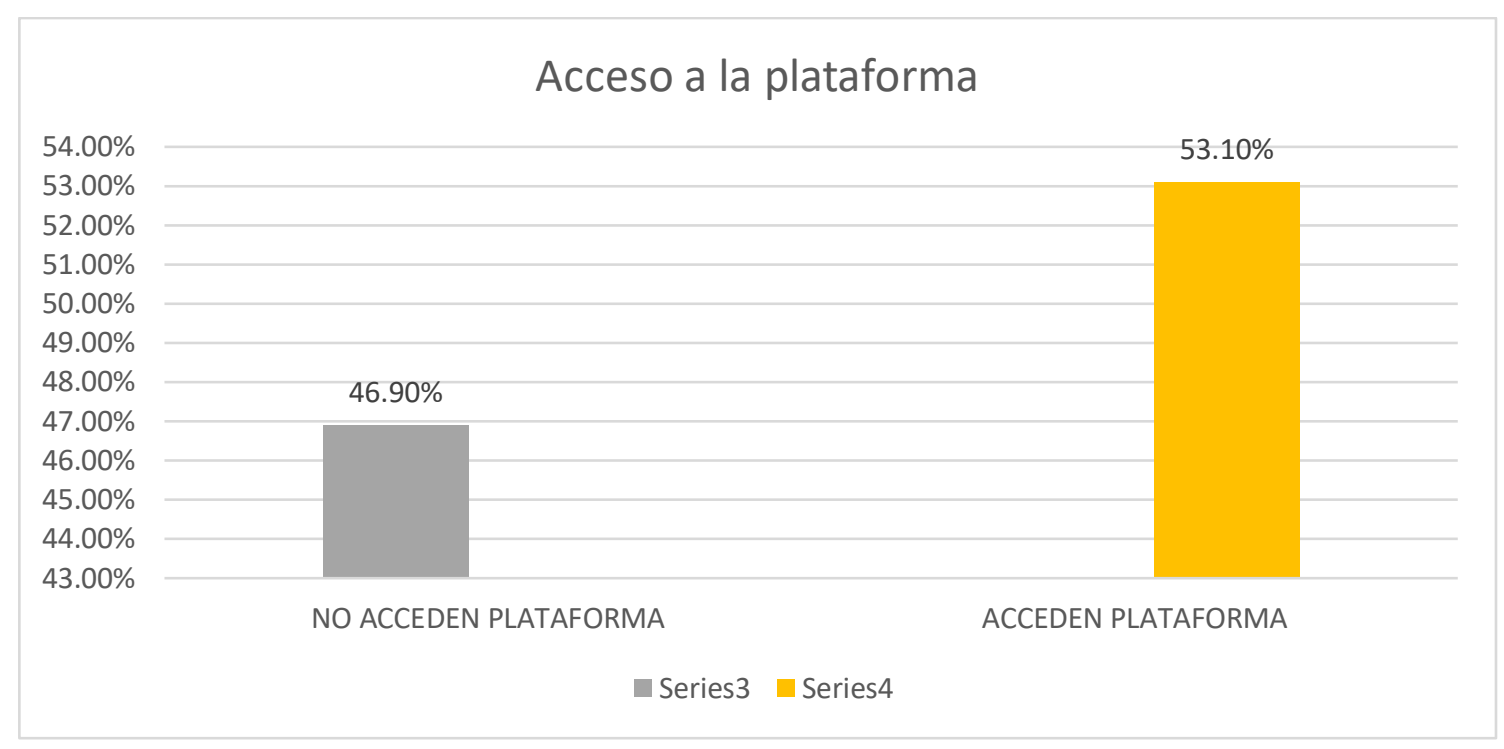

Figura 1. Porcentaje de acceso a plataforma

La figura 2 ilustra las aprobaciones del curso con 1456 bachilleres correspondiente al $35.75 \%$ quienes superan el $70 \%$ en sus evaluaciones de dominios y asistencias, y consecuentemente aprueban el curso, siendo el dato de mayor relevancia. Lo negativo es que reprueban 433 bachilleres, correspondiente al 10\% de participantes.

Para calcular las aprobaciones se consideraron los puntajes de cinco actividades curriculares de dominios por semana, durante 10 semanas, con un total de 150 actividades evaluadas. Adicionalmente se observó la interacción en el aula virtual por un mínimo del $70 \%$ de las actividades planificadas en los dominios. Las aulas virtuales estuvieron habilitadas durante 24 horas diarias por 7 días semanales para las actividades parciales de dominios.

Sobre aquellos bachilleres que se inscribieron en el curso, mediante la plataforma de la SENESCYT, pero luego se retiraron del mismo; este grupo tomó el cupo sin conocer previamente la metodología del curso (modalidad virtual y en línea) y los horarios de trabajo. Una vez que ingresaron a la plataforma, optaron por su retiro voluntario, sin registro de actividades de aprendizaje. Un aspecto determinante que lleva a tomar la medida de retirarse responde a las ofertas "facilistas" de cursos presenciales o semipresenciales en el mercado que "garantizan" la postulación a las carreras que se encuentran interesados.

También, manifiestan desconfianza en los procesos implementados por la SENESCYT, porque justamente en este curso, a última hora se modificaron las fechas de inicio a un período muy cercano al examen Ser Bachiller. Esta circunstancia no favoreció con el tiempo suficiente para su preparación, porque fue apenas de 36 días de lunes a domingo con un promedio de 7 horas diarias de dedicación casi exclusiva a su capacitación, lo que comúnmente se hace con un tiempo máximo de 4 horas diarias, en unos 63 días de lunes a viernes, en unas 12 semanas aproximadamente, con intervalos de descanso.

Otra de las razones por las que varios bachilleres se retiraron, responde a la falta de infraestructura tecnológica en los diferentes puntos del país. Así mismo, varios bachilleres viven y trabajan en sectores rurales lo que limita desarrollar las actividades a diario, como se programó en la plataforma. El encuentro se relaciona con el informe de que "el 36,0\% de 
los hogares a nivel nacional tienen acceso a internet (...). En el área urbana el 63,8\% de la población ha utilizado internet, frente al 38.0\% del área rural” (INEC, 2016, pág. 7 y 14).

Finalmente, existe un aspecto poco analizado, pero que es una realidad en los jóvenes comprendidos entre los 18 y 25 años, justamente en este rango de edades existen casos de quienes desean ingresar a las instituciones de educación superior, pero viven una movilidad marcada, que obliga a renunciar de forma inesperada a sus objetivos y aspiraciones de profesionalización. Pues se trasladan a otras ciudades, incluso al exterior, se cambian de sector de vivienda por el trabajo o por la necesidad de trabajo o porque forman una familia y adquieren otras responsabilidades.

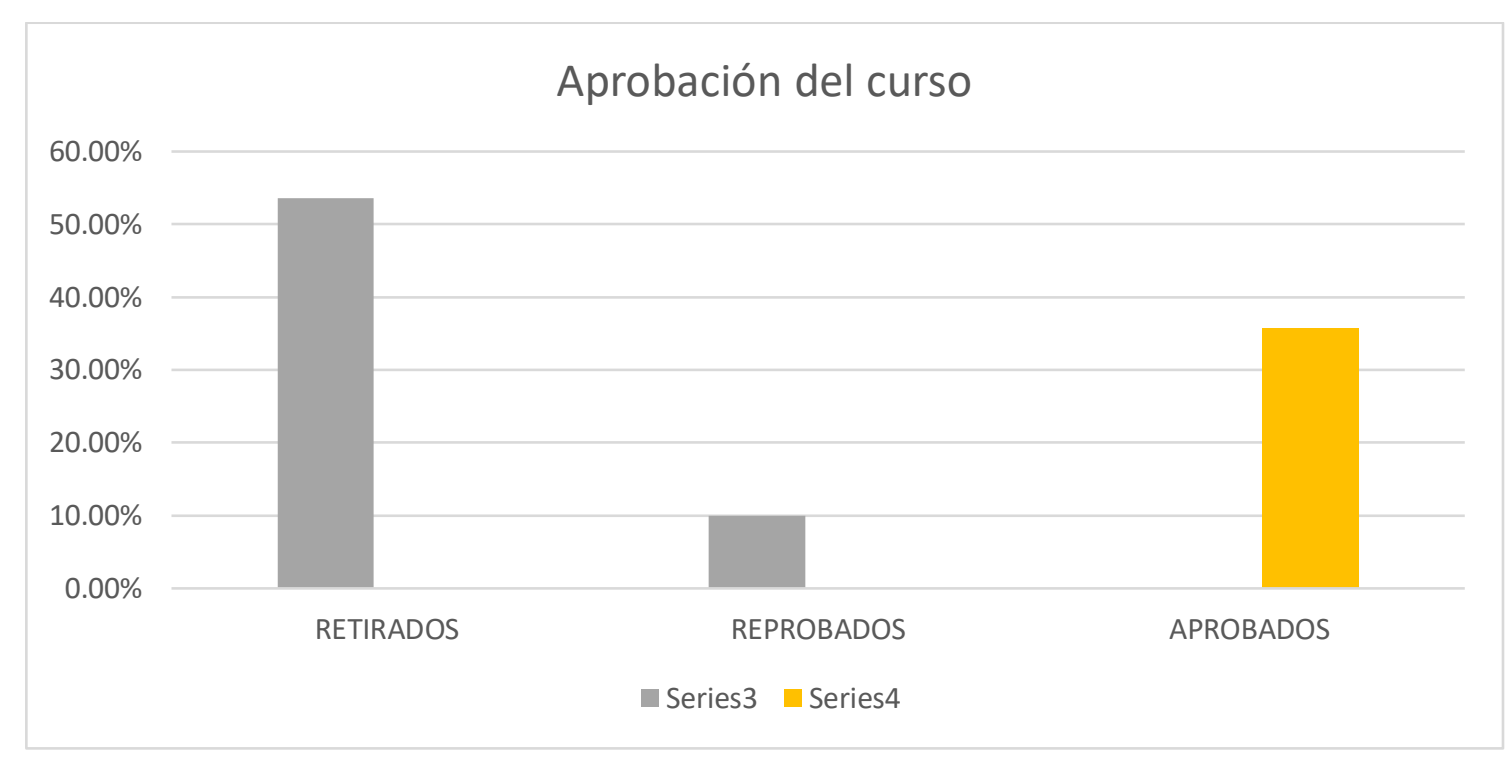

Figura 2. Aprobación del curso

El cuadro 2 sistematiza los indicadores de participación efectiva de bachilleres en el curso de nivelación en la categoría género, sobre lo que se analiza a 4073 participantes. Del total señalado son datos válidos para el análisis 2096 bachilleres (51.5\%), mientras que 1977 (48.5\%) son datos perdidos, y corresponde a aquellos que no respondieron la encuesta sobre su identidad de género.

Las aprobaciones por género, demuestran que son las mujeres quienes tienen mayor éxito en el curso, con un total de 786 bachilleres (69.1\%) del total de aprobados. Los varones solamente son 347, el 30.5\%. Mientras que 9 jóvenes aprobados, un (0.4\%), identifican su género con la opción otros. La tendencia de mayor aprobación de féminas está dada por la composición genérica de los participantes en el curso que fue de 4073 (100\%), de los cuales $1405(34.5 \%)$ se identifican como damas, frente a 682, (16.7\%) de caballeros, y otros con el $0.4 \%$, lo que da un $51.5 \%$ del total. El develamiento está corroborado con la afirmación que: "las mujeres (...) al momento de las evaluaciones o exámenes acuden más que los hombres al apoyo de los amigos, profesores o familiares cuando tienen dudas en los temas de estudio, están más conscientes del proceso enseñanza-aprendizaje que los hombres" (Sepúlveda, y otros, 2011, pág. 3). 


\begin{tabular}{lrrrr}
\hline Género & Aprobado & Reprobado & Retirado & Total \\
\hline Femenino & 786 & 178 & 441 & 1405 \\
Masculino & $69.1 \%$ & $65.2 \%$ & $64.4 \%$ & $67.0 \%$ \\
& 347 & 93 & 242 & 682 \\
Otros & $30.5 \%$ & $34.1 \%$ & $35.3 \%$ & $32.5 \%$ \\
& 5 & 2 & 2 & 9 \\
Total & $0.4 \%$ & $0.7 \%$ & $0.3 \%$ & $0.4 \%$ \\
& 1138 & 273 & 685 & 2096 \\
& $100.0 \%$ & $100.0 \%$ & $100.0 \%$ & $100.0 \%$ \\
\hline
\end{tabular}

Cuadro 2. Participación de bachilleres en el curso, por género

El cuadro 3 analiza la composición de bachilleres aprobados por etnias. En primer lugar se encuentra que la población mestiza es la de mayor participación en los distintos indicadores descritos del curso, porque aprueban 1 294, el (89.3\%) de beneficiarios. En segundo lugar de aprobación están los montubios con 58 personas $(4,0 \%)$, lo cual, sumado a otros indicadores como la procedencia por provincias y colegios, explica que la mayor población de bachilleres participantes en el curso proceden de la región litoral ecuatoriana.

Luego, pero muy lejanamente, se ubica la población autodeterminada como blanca, seguida de los indígenas, afrodescendientes, mulatos y otros, con porcentajes muy bajos y, que sumados dan un $7 \%$ de participantes, lo cual estadísticamente es poco significativo. El resultado evidencia y a la vez permite "(...) observar que existen diferencias en los promedios por auto identificación étnica, donde los grupos indígenas y afroecuatorianos mantienen menores promedios que los que se autoidentifican como mestizo/blanco" (Arévalo-Gross, 2018, pág. 132).

\begin{tabular}{lrrrr}
\hline \multicolumn{1}{c}{ Etnia } & Aprobado & Reprobado & Retirado & \multicolumn{1}{c}{ Total } \\
\hline Afrodescendiente & 24 & 11 & 72 & 105 \\
& $1.6 \%$ & $2.6 \%$ & $3.3 \%$ & $2.7 \%$ \\
Blanco & 37 & 16 & 59 & 112 \\
& $2.6 \%$ & $3.7 \%$ & $2.8 \%$ & $2.8 \%$ \\
Indígena & 19 & 2 & 27 & 48 \\
& $1.3 \%$ & $0.5 \%$ & $1.3 \%$ & $1.2 \%$ \\
Mestizo & 1294 & 363 & 1827 & 3484 \\
& $89.3 \%$ & $84.6 \%$ & $86.1 \%$ & $87.1 \%$ \\
Montubio & 58 & 28 & 96 & 182 \\
& $4.0 \%$ & $6.5 \%$ & $4.5 \%$ & $4.6 \%$ \\
Mulato & 15 & 8 & 29 & 52 \\
& $1.0 \%$ & $1.9 \%$ & $1.4 \%$ & $1.3 \%$ \\
Otro & 2 & 1 & 14 & 17 \\
& $0.1 \%$ & $0.2 \%$ & $0.7 \%$ & $0.4 \%$ \\
Total & 1449 & 429 & 2122 & 4000 \\
& $100.0 \%$ & $100.0 \%$ & $100.0 \%$ & $100.0 \%$ \\
\hline
\end{tabular}

Cuadro 3: Participación por identidad étnica

La composición de participantes aprobados (cuadro 4), al categorizar por discapacidad, quienes expresaron no tener discapacidad, pero aprueban el curso son 1446 (99.3\%) 
bachilleres, casi la totalidad de beneficiarios. Mientras que, quienes expresaron tener alguna discapacidad: "auditiva, física, intelectual, lenguaje, psicosocial y visual" (Dirección Nacional de Discapacidades (DND), 2019, pág. 3), y aprobaron, no superan el 1\% de bachilleres. El dato es inferior al porcentaje de discapacidades que en el 2019 es de $274 \%$, al dividir entre la población nacional, misma que según el Instituto Nacional de Estadísticas y Censos (INEC) es de 17379713 habitantes (INEC, 2019), con la cifra de 475747 personas con alguna discapacidad registrada en Consejo Nacional para la Igualdad de Discapacidades.

\begin{tabular}{lrrrr}
\hline Discapacidad & Aprobado & Reprobado & Retirado & Total \\
\hline Física & 5 & 2 & 3 & 10 \\
Ninguna & $0.3 \%$ & $0.5 \%$ & $0.1 \%$ & $0.2 \%$ \\
& 1446 & 429 & 2168 & 4043 \\
\multirow{2}{*}{ Psíquica } & & & & \\
& $99.3 \%$ & $99 . \%$ & $99.3 \%$ & $99.3 \%$ \\
Otra & 1 & 1 & 1 & 3 \\
\multirow{2}{*}{ Total } & $0.1 \%$ & $0.2 \%$ & $0,0 \%$ & $0.1 \%$ \\
& 4 & 1 & 12 & 17 \\
& $0.3 \%$ & $0.2 \%$ & $0,5 \%$ & $0.4 \%$ \\
& 1456 & 433 & 2184 & 4073 \\
& $100.0 \%$ & $100.0 \%$ & $100.0 \%$ & $100.0 \%$ \\
\hline
\end{tabular}

El cuadro 5 examina la participación por provincia. La estadística de matrícula y participación más significativa de bachilleres en el curso, al categorizar las 23 provincias que tienen presencia, destaca que Guayas es la de mayor representación 1453 (36.6\%), seguida de Manabí con 721, el (18.16\%) de jóvenes en tercer lugar, Pichincha con 569 (14. 33\%), en cuarto lugar, El Oro con 379 (9.5\%) y en quinto lugar Los Ríos con 199 (5\%).

La característica participativa de los bachilleres de las cinco provincias más representadas se puede atribuir a la conformación poblacional de jóvenes en edad de ingreso a la educación superior. Otro factor causal puede ser la cobertura de conectividad y una mayor difusión por parte de la SENESCYT en determinadas provincias. Las 18 provincias restantes tienen una participación poco importante.

El índice de aprobación del curso, al categorizar por provincias revela un comportamiento estadístico distinto a la frecuencia de matriculación. Las cuatro jurisdicciones de mejor rendimiento son: Tungurahua 15, Imbabura 29, Loja 44 y Azuay 14, pese a que su matrícula es muy baja. Mientras que, en las cinco provincias de mayor participación, aproximadamente uno de cada tres bachilleres matriculados aprueba la nivelación. Este análisis empírico coincide con (García \& Muñoz, 1999).

\begin{tabular}{|c|c|c|c|c|}
\hline Provincia & Aprobados & Reprobados & Retirados & Total \\
\hline Azuay & 14 & 2 & 21 & 37 \\
\hline Bolívar & 6 & 4 & 9 & 19 \\
\hline Cañar & 4 & 1 & 6 & 11 \\
\hline Carchi & 5 & 0 & 4 & 9 \\
\hline Chimborazo & 9 & 0 & 10 & 19 \\
\hline Cotopaxi & 3 & 1 & 15 & 19 \\
\hline El Oro & 148 & 41 & 190 & 379 \\
\hline Esmeraldas & 16 & 4 & 31 & 51 \\
\hline Galápagos & 1 & 0 & 2 & 3 \\
\hline Guayas & 463 & 147 & 843 & 1453 \\
\hline Imbabura & 29 & 5 & 28 & 62 \\
\hline
\end{tabular}




$\begin{array}{lrrrr}\text { Loja } & 44 & 14 & 44 & 102 \\ \text { Los Ríos } & 74 & 25 & 100 & 199 \\ \text { Manabí } & 286 & 93 & 342 & 721 \\ \text { Napo } & 1 & 0 & 4 & 5 \\ \text { Orellana } & 0 & 0 & 1 & 1 \\ \text { Pastaza } & 0 & 0 & 3 & 3 \\ \text { Pichincha } & 209 & 68 & 292 & 569 \\ \text { Santa Elena } & 48 & 10 & 81 & 139 \\ \text { Santo Domingo de los } & 42 & 8 & 77 & 127 \\ \text { Tsáchilas } & 1 & 0 & 5 & 6 \\ \text { Sucumbíos } & 15 & 2 & 13 & 30 \\ \text { Tungurahua } & 2 & 1 & 3 & 6 \\ \text { Zamora Chinchipe } & 1420 & 426 & 2125 & 3971 \\ \text { Total } & \text { Cuadro 5: Participación de bachilleres por provincia }\end{array}$

El cuadro 6 resume la matrícula y participación de bachilleres por cantones. Se priorizan para el análisis 44, en los que se evidencia la participación de mínimo 10 bachilleres en la nivelación, de entre 147 jurisdicciones beneficiarias. El primer aspecto relevante de territorialidad es que los 34 cantones que más participan en el curso son de la Región Litoral, 10 de la Región Sierra y, ninguno de la Amazonia y Galápagos. El orden de participación de bachilleres se correlaciona con la composición poblacional por regiones del Ecuador, sin embargo, hay una superioridad de bachilleres de poblaciones costaneras.

Los cinco cantones de mayor tasa de matrícula, en orden descendente son: Guayaquil con 1.215 bachilleres, el doble que Quito con 518, luego Machala con 214, en seguido por Portoviejo con 127 y Santo Domingo de los Tsáchilas con 114 bachilleres. Guayaquil tiene la mayor inscripción en la nivelación, con una tercera parte de bachilleres de todo el país, y más del doble de Quito, siendo el mayor beneficiario.

La tasa de aprobación cantonal, según la observación estadística reveló que de los 44 analizados, aprueban aproximadamente uno de cada tres bachilleres, esto es 1136, (35\%), mientras que reprueban 336 (10.35\%), y desertan 1774 (53 73\%), cifra muy alta. Los datos de base surgen de los 3246 bachilleres (100\%) matriculados en los cantones con más presencia de bachilleres. Los 726 bachilleres no analizados corresponden a 103 cantones, cuya presencia en la nivelación no supera a 9 sujetos matriculados.

\begin{tabular}{lrrrr}
\hline Cantón & Aprobado & Reprobado & Retirado & Total \\
\hline 24 de Mayo & 6 & 2 & 3 & 11 \\
Ambato & 10 & 2 & 11 & 23 \\
Arenillas & 5 & 2 & 8 & 15 \\
Buena Fe & 6 & 0 & 5 & 11 \\
Cayambe & 4 & 0 & 6 & 10 \\
Chone & 11 & 0 & 14 & 25 \\
Cuenca & 9 & 1 & 12 & 22 \\
Daule & 8 & 3 & 20 & 31 \\
DM. Quito & 190 & 64 & 264 & 518 \\
Durán & 21 & 13 & 49 & 83 \\
El Carmen & 12 & 1 & 10 & 23 \\
El Empalme & 3 & 2 & 7 & 12 \\
El Guabo & 5 & 2 & 18 & 25 \\
Esmeraldas & 10 & 3 & 21 & 34 \\
Guayaquil & 396 & 114 & 705 & 1215 \\
Huaquillas & 2 & 1 & 14 & 17 \\
\hline
\end{tabular}

Licencia Creative Commons Atribución 4.0 Internacional (CC BY 4.0)

Revista Cátedra, 3(2), pp. 17-34, mayo-agosto 2020. e-ISSN:2631-2875

https://doi.org/10.29166/10.29166/catedra.v3i2.2053 


\begin{tabular}{|c|c|c|c|c|}
\hline Ibarra & 19 & 4 & 21 & 44 \\
\hline Jipijapa & 12 & 1 & 22 & 35 \\
\hline La Concordia & 4 & 0 & 9 & 13 \\
\hline La Libertad & 21 & 5 & 41 & 67 \\
\hline La Troncal & 3 & 1 & 6 & 10 \\
\hline Loja & 23 & 13 & 27 & 63 \\
\hline Machala & 100 & 23 & 91 & 214 \\
\hline Mejía & 4 & 0 & 7 & 11 \\
\hline Milagro & 9 & 4 & 20 & 33 \\
\hline Montecristi & 19 & 10 & 26 & 55 \\
\hline Naranjito & 3 & 1 & 6 & 10 \\
\hline Otavalo & 6 & 0 & 4 & 10 \\
\hline Pasaje & 15 & 5 & 32 & 52 \\
\hline Pedernales & 2 & 1 & 11 & 14 \\
\hline Piñas & 2 & 2 & 7 & 11 \\
\hline Portoviejo & 46 & 18 & 63 & 127 \\
\hline Quevedo & 26 & 8 & 40 & 74 \\
\hline Riobamba & 9 & 0 & 9 & 18 \\
\hline Rocafuerte & 10 & 4 & 6 & 20 \\
\hline Rumiñahui & 8 & 3 & 12 & 23 \\
\hline Salinas & 8 & 2 & 11 & 21 \\
\hline Santa Ana & 7 & 2 & 6 & 15 \\
\hline Santa Elena & 19 & 3 & 29 & 51 \\
\hline Santa Rosa & 15 & 4 & 12 & 31 \\
\hline Santo Domingo & 38 & 8 & 68 & 114 \\
\hline Sucre & 5 & 1 & 8 & 14 \\
\hline Valencia & 2 & 2 & 6 & 10 \\
\hline Vinces & 3 & 1 & 7 & 11 \\
\hline Total & 1136 & 336 & 1774 & 3246 \\
\hline No analizados & & & & 726 \\
\hline Matriculados & & & & 3972 \\
\hline
\end{tabular}

El cuadro 7 contiene la data de matrícula de bachilleres por tipo de institución educativa de procedencia. Al respecto 1568 beneficiarios, (74.49\%) son de planteles públicos, 363 $(17.24 \%)$ son de planteles municipales, 154 (7.32\%) son de planteles particulares y apenas $20(0.95 \%)$ de origen fisco-misional (financiamiento público-privado).

La composición de beneficiarios aprobados y no aprobados, con relación a los tipos de institución educativa de procedencia del bachiller, corresponde a 845 (73.9\%) bachilleres son de origen público. Un importante grupo de 197 (17.2\%) jóvenes son de planteles privados en segundo lugar; 90 (7.9\%) de bachilleres son de origen fisco-misional en tercer lugar, y; solamente 10 son de unidades educativas municipales, para un total de aprobados de 1143 (100\%).

\begin{tabular}{lrrrr}
\hline & Aprobado & Reprobado & Retirado & Total \\
\hline Fisco misional & 91 & 19 & 44 & 154 \\
Municipal & 10 & 3 & 7 & 20 \\
Privado & 197 & 53 & 113 & 363 \\
Público & 845 & 199 & 524 & 1568 \\
Total & 1143 & 274 & 688 & 2105 \\
\hline
\end{tabular}

Cuadro 7. Matrícula y aprobación por institución educativa 
El cuadro 8 condensa los promedios generales de notas de las evaluaciones aplicadas por dominio. Las evaluaciones tienen tareas, deberes y ejercicios que los bachilleres respondieron mediante cuestionarios aplicados en las aulas virtuales, cuyas notas están alojadas en la plataforma de la UCE.

Los promedios de las notas logradas por los bachilleres en la plataforma son sobre 15 puntos semanales por 10 semanas, para un total de 150. Los puntajes se ponderaron a 1.000 puntos, para analogar al puntaje del examen Ser Bachiller.

Al analizar los promedios de aprobación del curso, con el modelo del SNNA, el más alto corresponde a dominio abstracto con 905. 39/1 000 puntos, y el más bajo a un dominio social con 812.12/1.000. El promedio general de los aprobados en todos los dominios es de 868. 55/1.000 puntos, lo que evidencia que los bachilleres beneficiarios obtuvieron notas consideradas dentro del rango de aprobación del examen Ser Bachiller, consecuentemente tienen opciones de postular a carreras universitarias estatales.

El promedio general de notas, por dominio, logradas en el curso, se observa en el dominio lingüístico con 588/600 y el más bajo en el dominio matemático con 493/600. En general los promedios de dominios considerando aprobados, reprobados y retirados es de 550/600 puntos que es un resultado global regular.

La evaluación se desarrolló como la última etapa del proceso curricular, para observar el comportamiento de los bachilleres sobre los conocimientos, habilidades, destrezas y capacidades cognitivas para la resolución eficaz del examen Ser Bachiller. Los instrumentos virtuales utilizados fueron: ejercicios, deberes, tareas, simulaciones, así como participación en los foros de interacción y participaciones en las conferencias virtuales. Un valioso indicador de evaluación fue la participación interactiva de los bachilleres en las aulas virtuales, porque de esta manera se miró el grado de preocupación y compromiso con su propia preparación para el examen, más allá del puntaje objetivo, (Martínez, 2015).

Los datos de notas logradas por aula y dominio sirvieron para valorar el progreso de los puntajes de trabajo y de progreso semanalmente. Con este diagnóstico permanente se pudo reforzar a aquellos bachilleres que necesitaron, y a la misma vez alentar a quienes tenían mejores puntajes.

Al final se puede extrapolar que los resultados de las evaluaciones, pese a la modalidad en línea, fueron bastante significativos, ya que 3 de cada cinco bachilleres, un $62 \%$ superan satisfactoriamente el 70\% mínimo para aprobación, como promedio de todas las evaluaciones y participación.

\begin{tabular}{lrrrr}
\hline \multicolumn{1}{c}{ Dominios } & $\begin{array}{c}\text { Promedio } \\
\text { General/15 }\end{array}$ & $\begin{array}{c}\text { Promedio General } \\
\text { /600 }\end{array}$ & $\begin{array}{c}\text { Promedio } \\
\text { Aprobados /150 }\end{array}$ & $\begin{array}{c}\text { Promedio } \\
\text { Aprobados/1000 }\end{array}$ \\
\hline Abstracto & 86.28 & 575 & 135.81 & 905.39 \\
Científico & 86.39 & 576 & 135.10 & 900.68 \\
Lingǘstico & 88.16 & 588 & 133.84 & 892.24 \\
Matemático & 73.88 & 493 & 135.13 & 900.88 \\
Social & 77.65 & 518 & 121.82 & 812.12 \\
Total & 82.47 & 550 & 651.41 & 868.55 \\
\hline
\end{tabular}

Cuadro 8. Resumen promedios evaluaciones por dominio 


\section{Conclusiones}

Los principales descubrimientos que se logran en el estudio, mismos que responden a las preguntas de investigación propuestas, son los siguientes:

El índice de los bachilleres retirados del curso es ligeramente superior que de los participantes permanentes. Los porcentajes de participación-deserción bordean el 50\% del total. Entre los principales factores causales para la deserción constan la desconfianza con las variaciones de los procesos planificativos del curso por la SENESCYT y la deficiente infraestructura tecnológica y comunicativa en los sectores periféricos de ciudades y sobre todo rurales.

Al observar la promoción de bachilleres participantes del curso de nivelación general, es muy superior el porcentaje de bachilleres que aprueban. Contrariamente, el porcentaje de aquellos que reprueban es muy bajo, pero también representativo, porque implica la afectación a la inversión pública del Estado, y a la vez un menor acceso al derecho de ingreso a las IES, especialmente de sectores marginados.

La inclusión por género en el curso de nivelación, se manifiesta en que el mayor porcentaje de participación y aprobación corresponde a las mujeres, quienes superan con más del doble a los varones. En la encuesta no se identificaron géneros que se nombren taxativamente, sino solamente en la categoría otros, pero en un porcentaje no significativo. Sin embargo, los datos perdidos son casi similares a la data válida.

La inclusión por etnias, expresa que hay un porcentaje muy alto de participación y aprobación del curso en el grupo que se auto define como mestizo. El resto de las etnias constantes en la encuesta no superan el un décimo del total e incluyen en orden a los montubios en segundo lugar, luego blancos, e indígenas afrodescendientes. La explicación de los montubios en el segundo lugar se debe a que la mayor participación fue de bachilleres costeños.

En cuanto a la inclusión por discapacidad, la data demuestra que los bachilleres sin ninguna discapacidad participan y aprueban el curso en casi el porcentaje total. Mientras que la participación de los bachilleres con alguna discapacidad es casi nula. Este fenómeno puede deberse a que la modalidad de la capacitación virtual y en línea no atiende a las necesidades educativas especiales, lo que implica que procesos posteriores deben atender a este sector.

En cuanto a la participación de bachilleres por provincias. La inclusión por territorialidad, indica que hay mayoritariamente una participación de las costaneras. En el grupo de mayor aprobación constan cuatro, también de la región litoral, una de la sierra y ninguna del oriente. Contrariamente, la aprobación del curso por provincias tiene a cuatro de la sierra en los primeros lugares, pese a que su presencia es mínima, lo cual explica en cierta medida, la heterogeneidad en la calidad educativa y la atención del Estado en el contexto nacional.

En cuanto a la participación de los bachilleres por cantones, en el curso participan 147, más de la mitad de los 209 cantones distribuidos en las 23 provincias, ya que de Morona Santiago no hubo presencia. La mayor cantidad de los cantones son de la costa, lo cual se relaciona también con que cuatro cantones entre cinco de mayor participación son litorales. Este hallazgo se correlaciona con la demografía nacional. La participación tiene una supremacía de provincias y cantones costaneros en el curso. En el porcentaje de aprobación por cantones, se descubrió que un tercio de bachilleres aprueban el cuso, un décimo reprueban y la mitad desertan. Se evidencia que la inclusión territorial es relativa, pues, no todos los cantones y provincias participan en igualdad de condiciones y porcentajes poblacionales. 
En inclusión por territorialidad de instituciones educativas, se manifiesta que dos quintos de bachilleres participantes son de planteles públicos (fiscales y municipales) y un décimo de particulares, como lo más representativo. Las aprobaciones varían, ya que las tres cuartas partes son de planteles oficiales (fiscales) en primer lugar, luego de particulares los dos quintos, finalmente un décimo aprueba de planteles fisco-misionales. Los jóvenes con mayor necesidad de capacitación para el examen Ser Bachiller son de colegios estatales, en comparación a los particulares y fisco-misionales.

Los promedios de notas logradas en la aprobación del curso reflejan que el puntaje promedio final de los dominios tratados, en el caso de ratificarse en el examen Ser Bachiller, proporcionarán opciones a los bachilleres para aprobar y postular a carreras universitarias, con cierta facilidad. Siempre que los bachilleres beneficiarios, consideren las recomendaciones de la Orientación Vocacional y Profesional (OVP) tratada en el curso.

Las notas de aprobación del curso pueden deberse al tiempo de gestión académica, muy corto e intensivo, con que se capacitó a bachilleres. Solamente 36 días laborados, de lunes a domingo. Un tiempo aproximado de siete horas diarias de participación en aula virtual, sin días de descanso y recuperación.

\section{Agradecimientos}

Al equipo docente, técnico y administrativo que participó en el cuso de nivelación general modalidad virtual, facilitado a bachilleres a nivel nacional, en los meses de mayo a julio de 2019, mediante convenio entre la Empresa Pública de Bienes y Servicios UCE PROYECTOS EP, con la SENESCYT.

\section{Bibliografía}

Aceves, F. (1997). La territorialidad. Punto nodal en la intersección espacio urbanoprocesos de comunicación-movimiento social. Comunicación y Sociedad (OECS, Universidad de Guadalajara(30), pp. 275-301.

Arévalo-Gross, J. (2018). La educación en Ecuador: logros alcanzados y nuvos desafíos. Resultados educativos 2017- 018. Quito: Instituto Nacional de Evaluación Educativa.

Asamblea Nacional. (2011). Ley Orgánica de Educación Intercultural. Quito: Asamblea Nacional.

Cociña, M. (07 de 06 de 2013). https://ciperchile.cl. Obtenido de https://ciperchile.cl/2013 /06 /07/cinco-argumentos-contra-la-meritocracia/

Dirección Nacional de Discapacidades (DND). (2019). www.salud.gob.ec. Obtenido de https://www.salud.gob.ec/direccion-nacional-de-discapacidades/

Escuela Politécnica Nacional. (18 de Diciembre de 2019). www.epn.edu.ec. Obtenido de https: //www.epn.edu.ec/sistema-nacional-de-nivelacion-y-admision-snna/

Farrell, G., Trillón, S., y Soto, P. (1999). Farrell, Gilda; La competitividad territorial: construir una estrategia de desarrollo territorial con base en la experiencia de leader. Innovación en el Medio Rural, Cuaderno No. 6 - Fascículo 1. Bruselas: Observatorio Europeo leader. 
García, Á., y Muñoz, J.-M. (1999). El factor territorial en la política educativa: ¿Es posible una fundamentación pedagógica? Universidad de Salamanca, 11, pp. 177-189.

García, E. (2007). El "abandono" en cursos de e-learning: algunos aprendizajes para nuevas propuestas. Revista Iberoamericana de Educación, 44(3), pp. 2-16.

Haro, O. (2018). Relación del examen nacional para la educación superior -ENES- y la vocación docente con el rendimiento académico del alumnado de la Facultad de Filosofía, Letras y Ciencias de la Educación de la Universidad Central del Ecuador. Alicante-España: Universidad de Alicante.

Inclusión Educativa. (2016). www.inclusioneducativa.org. Obtenido de http:// www.inclusion educativa.org/ise.php?id=1

INEC. (2016). www.ecuadorencifras.gob.ec. Obtenido de https://www.ecuadorencifras.gob .ec/documentos/web-inec/Estadisticas_Sociales/TIC/2016/170125.Presentacion _Tics_2016.pdf

INEC. (15 de 12 de 2019). www.ecuadorencifras.gob.ec. Obtenido de https://www.ecuador encifras.gob.ec/estadisticas/

Leiva, J.-J. (2013). De la integración a la inclusión: evolución y cambio en la mentalidad del alumnado universitario de educación especial en un contexto universitario español. Actualidades Investigativas en Educación, 13(3), pp. 1-26.

Martínez, N. (13 de Marzo de 2015). http://www.redicces.org.sv/jspui/handle/10972/2037. Obtenido de http://www.redicces.org.sv/jspui/bitstream/10972/2037/1/2.\%20 Evaluacion\%20de\%20los\%20aprendizajes\%20en\%20la\%20educacion\%20virtua l.pdf

Otzen, T. y Manterola, C. (2017). Técnicas de Muestreo sobre una Población a Estudio. Int. J. Morphol., 35(1), pp. 227-232.

Pérez-Castro, J. (2019). La inclusión de los estudiantes con discapacidad en dos universidades públicas mexicanas. Innovación Educativa, 19(79), pp. 145-170.

SENESCYT. (2019). Reglamento del Sistema Nacional de Nivelación y Admisión SNNA. Quito: Registro Oficial.

SENESCYT-Empresa Pública UCE. (2019). Términos de referencia para contratar la prestación del servicio de nivelación general mediante un curso en modalidad virtual a nivel nacional, para las y los aspirantes que postularon en el primer semestre 2019 y no obtuvieron un cupo a través del sistem. Quito: SENESCYT-UCE.

Sepúlveda, J., López, M., Torres, P., Luengo, J., Montero, E., y Contreras, E. (2011). Diferencias de género en el rendimiento académico y en el perfil de estilos y de estrategias de aprendizaje en estudiantes de Química y Farmacia de la Universidad de Concepción. Revista Estilos de Aprendizaje, 7(7), pp. 1-16. 
SNNA. (11 de Diciembre de 2011). www.educacionsuperior.gob.ec. Obtenido de https:// www.educacionsuperior.gob.ec/wp-content/uploads/downloads/2012/07 /SNNA _presenta ción.pdf

Villalobos, 0. (26 de 09 de 2015). http://journalrural.com. Obtenido de http://journalrural .com /enfoque-territorial/\#sthash.00a2hWtN.dpbs

\section{Autores}

OSWALDO HARO-JÁCOME Doctor PhD. en Investigación Educativa obtenido en la Universidad de Alicante-España; Magister en Educación; Doctor en Investigación Socioeducativa; Licenciado en Filosofía y Ciencias Socioeconómicas; Profesor Normalista.

Docente titular de la Universidad Central del Ecuador, actualmente en la Carrera de Pedagogía de la Historia y las Ciencias Sociales de la Universidad Central del Ecuador; Exdirector del Instituto Superior de Extensión Universitaria; director de la Carrera Plurilingüe; Ex-Docente titular de la Universidad de las Fuerzas Armadas ESPE; Ex-Docente Colegio Municipal Sebastián de Benalcázar y Ex-Vicerrector de la Unidad Educativa Municipal Eugenio Espejo.

HECTOR SIMBAÑA-CABRERA Doctor PhD. en Investigación Educativa obtenido en la Universidad de Alicante-España; Maestría en Docencia Universitaria y Administración Educativa; Licenciado en Ciencias de la Educación Profesor de Enseñanza Media. Especialización Biología y Química.

Docente titular de la Universidad Central del Ecuador, actualmente en la Carrera de Pedagogía de la Historia y las Ciencias Sociales de la Universidad Central del Ecuador; ExSubdecano de la Facultad de Filosofía, Letras y Ciencias de la Educación; Exdirector del Departamento de Extensión Universitaria; Coordinador de las Prácticas pre profesionales de la Universidad Central del Ecuador; Miembro del equipo técnico de la Coordinación General de Vinculación con la sociedad de la UCE.

JUAN AGUILAR-POAQUIZA Doctor en Gestión Empresarial de la Universidad Mayor Nacional de San Marcos Lima-Perú, Ingeniero de Empresas y Master en Finanzas.

Docente titular y Director de Vinculación con la Sociedad de la Escuela Superior Politécnica de Chimborazo. Experiencia docente desde el año de 2001 en el área financiera y formador de conocimientos en las áreas de Portafolio de Inversiones y Mercado de Capitales. En el sector privado, gerente de comercialización de la Asociación de Granos y Gramíneas del Ecuador. 